\title{
Theory of mind in a first-episode psychosis population using the Hinting Task
}

\section{Lindgren, Maija}

2018-05

Lindgren , M , Torniainen-Holm , M , Heiskanen , I, Voutilainen , G, Pulkkinen , U , Mehtälä , T , Jokela , M , Kieseppä , T , Suvisaari , J \& Therman , S 2018 , ' Theory of mind in a first-episode psychosis population using the Hinting Task ' , Psychiatry Research , vol. 263 , pp. 185-192 . https://doi.org/10.1016/j.psychres.2018.03.014

http://hdl.handle.net/10138/300095

https://doi.org/10.1016/j.psychres.2018.03.014

cc_by_nc_nd

acceptedVersion

Downloaded from Helda, University of Helsinki institutional repository.

This is an electronic reprint of the original article.

This reprint may differ from the original in pagination and typographic detail.

Please cite the original version. 


\section{Accepted Manuscript}

Theory of mind in a first-episode psychosis population using the Hinting Task

Maija Lindgren, Minna Torniainen-Holm , Inkeri Heiskanen, Greta Voutilainen, Ulla Pulkkinen, Tuukka Mehtälä ,

Markus Jokela , Tuula Kieseppä , Jaana Suvisaari , Sebastian Therman

PII: S0165-1781(17)32202-3

DOI: 10.1016/j.psychres.2018.03.014

Reference: PSY 11246

To appear in:

Psychiatry Research

Received date:

4 December 2017

Revised date:

5 March 2018

Accepted date:

5 March 2018

Please cite this article as: Maija Lindgren, Minna Torniainen-Holm, Inkeri Heiskanen, Greta Voutilainen, Ulla Pulkkinen, Tuukka Mehtälä, Markus Jokela, Tuula Kieseppä, Jaana Suvisaari, Sebastian Therman, Theory of mind in a first-episode psychosis population using the Hinting Task, Psychiatry Research (2018), doi: 10.1016/j.psychres.2018.03.014

This is a PDF file of an unedited manuscript that has been accepted for publication. As a service to our customers we are providing this early version of the manuscript. The manuscript will undergo copyediting, typesetting, and review of the resulting proof before it is published in its final form. Please note that during the production process errors may be discovered which could affect the content, and all legal disclaimers that apply to the journal pertain. 


\section{Highlights}

- There were impairments in Theory of Mind (ToM) in early psychosis.

- Deficits in ToM largely overlapped with deficits in general cognitive processes.

- Specific deficits in ToM in schizophrenia were independent from general cognition.

- Patients with other psychotic disorders did not differ from controls.

- The internal consistency of the Hinting Task was modest.

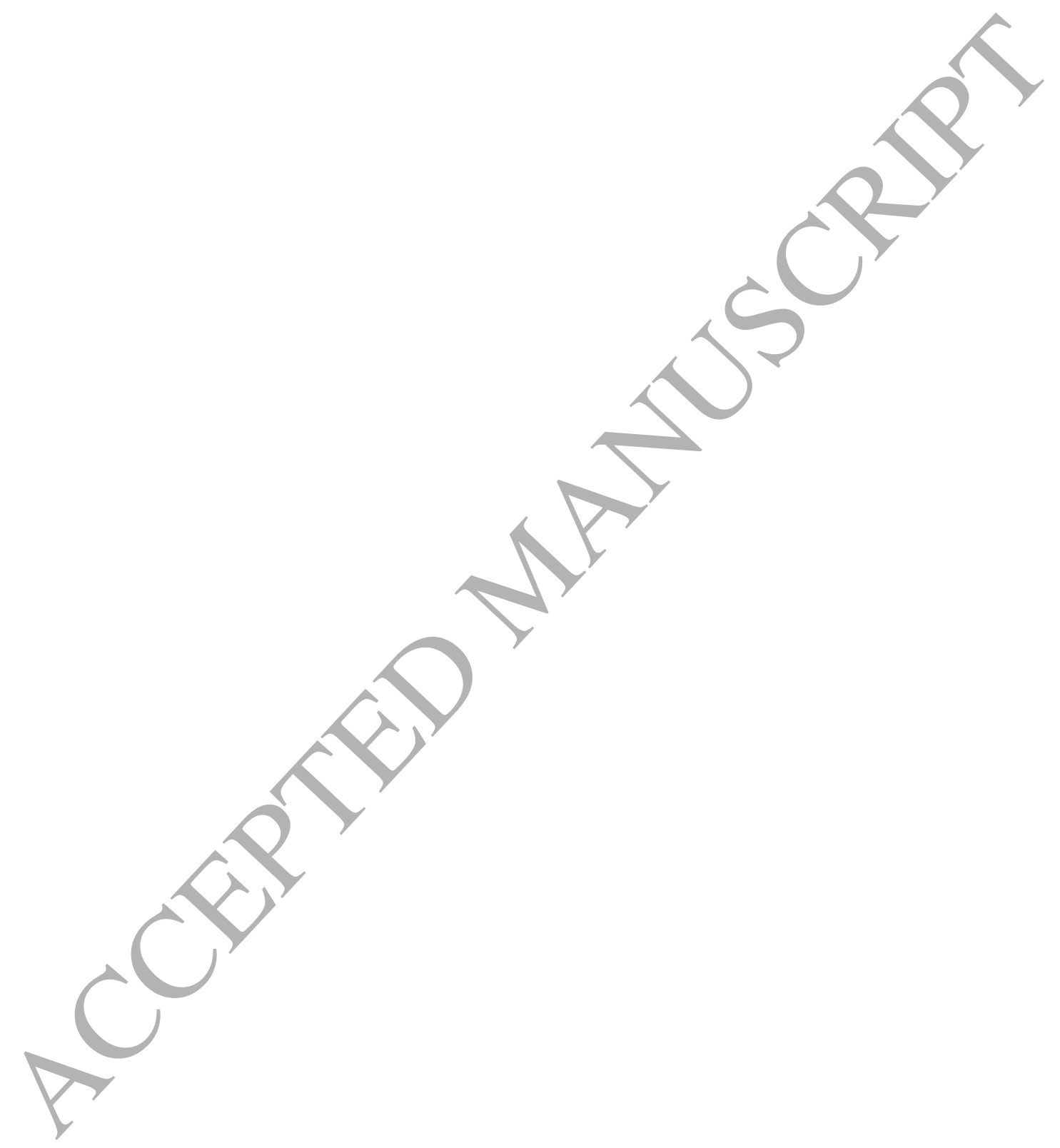




\section{Theory of mind in a first-episode psychosis population using the Hinting Task}

Maija Lindgren ${ }^{\mathrm{a} *}$, Minna Torniainen-Holm ${ }^{\mathrm{a}}$, Inkeri Heiskanen ${ }^{\mathrm{b}}$, Greta Voutilainen ${ }^{\mathrm{c}}$, Ulla Pulkkinen $^{\text {ad }}$, Tuukka Mehtälä ${ }^{\mathrm{e}}$, Markus Jokela ${ }^{\mathrm{f}}$, Tuula Kieseppä ${ }^{\mathrm{ag}}$, Jaana Suvisaari ${ }^{\text {a }}$, Sebastian Therman $^{\text {a }}$

${ }^{a}$ Mental Health Unit, National Institute for Health and Welfare (THL). PO Box 30, FIN-00271, Helsinki, Finland

${ }^{\mathrm{b}}$ Helsinki Deaconess Institute, Helsinki, Finland

${ }^{\mathrm{c}}$ Social Services and Health Care, City of Helsinki, Aurora Hospital, Helsinki, Finland

${ }^{\mathrm{d}}$ Department of Psychiatry, Royal College of Surgeons in Ireland, Dublin, Ireland

${ }^{\mathrm{e}}$ Department of Psychiatry, Hospital District of Helsinki and Uusimaa, Hyvinkää Hospital Area, Kellokoski, Finland

${ }^{\mathrm{f}}$ Department of Psychology and Logopedics, Faculty of Medicine, Helsinki University, Helsinki, Finland

${ }^{\mathrm{g}}$ Department of Psychiatry, University of Helsinki and Helsinki University Hospital, Helsinki, Finland

* Corresponding author. maija.lindgren@thl.fi. Mental Health Unit, National Institute for Health and Welfare, PO Box 30, FIN-00271 Helsinki, Finland. Tel.: +358 29524 7015; fax: +358 29524 7155 .

\section{Abstract}

Deficiencies in theory of mind (ToM) are common in psychosis and may largely explain impaired social functioning. Currently, it is unclear whether impairments in ToM are explained by the more general cognitive/deficits related to psychosis or whether ToM is impaired in psychosis independently of other cognitive deficits. This study examined ToM using the Hinting Task in young adults $(n=66)$ with first-episode psychosis and matched controls $(n=62)$. The participants were administered a broad neuropsychological assessment. Participants with psychosis performed worse than controls on the Hinting Task. However, $75 \%$ of the variance between the groups was explained by general cognitive deficits, especially impaired processing speed and episodic memory. 
Hinting Task performance of the best functioning patient group did not differ from that of the control group. When the psychosis group was divided according to diagnosis, the Hinting Task difference between individuals with schizophrenia and controls remained significant even when general cognitive performance was controlled for, suggesting specific verbal ToM deficits in schizophrenia. In contrast, those with other psychotic disorders did not differ from controls. Our results suggest that ToM deficits can be seen in early phases of psychotic disorders, schizophrenia in particular, and are partly independent of other cognitive functions.

Keywords: social cognition; theory of mind; Hinting Task; first-episode psychosis; schizophrenia; cognition

\section{Introduction}

Social cognition can be defined as perception and interpretation of and responding to other people's thoughts and actions. According to one division, social cognition consists of emotional processing, social perception, social knowledge, attributional styles, and theory of mind (ToM) (Green et al., 2008). ToM can be defined as the ability to understand the mental states of others (Green et al., 2008). In order to comprehend the way the other people act, one has to observe their point of view and - based on social cues and context - make assumptions of their mental states. This “mentalizing" enables prediction and understanding of other people's actions. Through for instance maladaptive interpretations and reactions and social withdrawal, deficits in these abilities may affect social behavior and social functioning in everyday interactions (Green et al., 2015). Social functioning in the community may therefore rely on intact social cognition or ToM. 
Among other cognitive deficits, impaired social cognition and especially ToM is often a part of a psychotic disorder (Bora and Pantelis, 2013). Individuals with psychosis may present with inappropriate reactions in social situations, concrete interpretation of speech, and decreased empathy (Green et al., 2015). Deficits in the processing of social information are especially common in schizophrenia (Bora et al., 2009) as well as in first-episode psychosis (FEP) (Bora and Pantelis, 2013; Healey et al., 2016) and even in individuals at ultra-high risk for psychosis (Van Donkersgoed et al., 2015). These kinds of deficits may cause social withdrawal, misinterpretations in social interaction, and other impaired social functioning (Green et al., 2015), and may therefore have a strong impact on the everyday life of the individual with a psychotic disorder.

Studies show that deficits in everyday functioning of patients with psychosis can partially be explained by neurocognition (especially verbal memory, executive function, and vigilance) (Green et al., 2000). However in another meta-analysis, social cognition was more strongly associated with functional outcome than neurocognition (Fett et al., 2011). In addition to independently predicting level of functioning, social cognition may mediate the association between other cognitive domains and functioning (Addington et al., 2010; Schmidt et al., 2011). Deficits in ToM have also been used to explain psychotic symptoms (Bentall et al., 2014). In the context of having difficulties inferring the beliefs and intentions of others and distinguishing between subjective beliefs and reality, for instance paranoia has been linked to "hypermentalizing" or over-attributing intention to others (Brüne, 2005; Green et al., 2015). In addition, the ability to understand others may affect the relationship with the treating personnel. Therefore, early identification of ToM deficits is essential to the therapeutic alliance in rehabilitation of psychosis (Green et al., 2008).

Performance in tasks assessing ToM correlates with those assessing other cognitive abilities, such as general intelligence, memory, and executive function (Bora et al., 2009). It is therefore possible that ToM deficits only reflect deficits in other specific cognitive deficits and general cognitive decline related to psychosis, rather than being independently related to psychosis. 
According to a recent review, specific deficits in ToM in FEP are not completely explained by general cognitive decline (Healey et al., 2016). Another review suggested that general cognitive deficits contribute to ToM impairment especially in the stable phase of psychotic illness (Bora et al., 2009). Social cognition and other cognitive functions appear to be partly overlapping, but deficiencies in a single area are also possible (Green et al., 2008). Further, social cognition and nonsocial cognition seem largely distinct at behavioral and neural level (Green et al., 2015).

In this study, the Hinting Task (Corcoran et al., 1995) was used to measure ToM abilities among young adults after their first psychiatric contact for psychosis. The Hinting Task has been found to be a psychometrically good social cognition measure in schizophrenia (Pinkham et al., 2016) and FEP populations (Ludwig et al., 2017), although psychometric problems (e.g., ceiling effects) have been noted also with this instrument. With short vignettes of everyday situations, the Hinting Task assesses the ability to conclude, from indirect speech, what another person really means. As ToM can be divided into "mental state decoding" (or affective ToM) and "mental state reasoning" (or cognitive ToM), with different associations to social functioning (Bora et al., 2006; McGlade et al., 2008), the Hinting Task specifically measures the latter, "mental state reasoning".

Neuropsychological assessment for controlling the role of general cognition in ToM has often been scarce and it is unclear whether ToM is impaired in psychosis independently of other cognitive deficits. We wanted to explore 1) the possible difference in ToM between FEP patients and controls, 2) whether diagnostic group (schizophrenia versus other psychotic disorders) and level of functioning are associated with ToM, and 3) to what extent these differences are explained by general cognitive performance. 


\section{Methods}

\subsection{Participants and study procedure}

The FEP group consisted of adult patients from hospitals and outpatient clinics of the City of Helsinki and Helsinki University Hospital participating in the Helsinki Psychosis Study (Keinänen et al., 2015; Lindgren et al., 2017), with first psychiatric treatment contact for psychosis. The participants were interviewed with the Brief Psychiatric Rating Scale (BPRS), Expanded version 4.0 (Ventura et al., 1993) as soon as possible after they had commenced treatment and were able to provide informed consent as judged by the treating personnel. Psychosis was defined as a score $\geq 4$ (moderate or higher) in unusual thought content or hallucinations, representing symptoms at the psychotic level. Exclusion criteria were psychotic disorders that were substance-induced or caused by a general medical condition. The baseline study also included questionnaires, magnetic resonance imaging (MRI), and laboratory assessments (blood and stool analyses). The study protocol included follow-ups after two and twelve months with both the BPRS and the Structured Clinical Interview for the DSM-IV, Research Version (SCID-I/P) (First et al., 2002).

Age- and gender-matched control participants from the same catchment area (towns of Helsinki, Vantaa, and Espoo and several smaller municipalities around them) were recruited from the Finnish Population Information System (a national register containing basic information about all Finnish citizens) and were assessed at baseline and at twelve months. The exclusion criteria were lifetime psychotic disorder, any condition preventing MRI, and chronic neurological or endocrinological diseases, the latter criteria being related to the other aspects of the overall research project. Other mental health problems were not an exclusion criterion.

In this study, we utilized data from baseline and from the two-month follow-up. In addition, diagnostic information based on the 12 month follow-up was used to obtain more reliable diagnoses, as for example diagnosis of schizophreniform disorder was often provisional at the twomonth follow-up. 
All the participants gave written informed consent to participation. The study protocol was approved by the Ethics Committee of the Hospital District of Helsinki and Uusimaa and by the institutional review boards of University of Helsinki and the National Institute for Health and Welfare, Finland. The study was carried out in accordance with the sixth version of the Declaration of Helsinki.

\subsection{Measures}

Cognitive testing included measures from the Wechsler Adult Intelligence Scale, Wechsler Memory Scale, and Trail Making Tests versions A and B, among others (Supplementary Table 1). The testing was part of the baseline assessment in controls and the two-month follow-up assessment in FEP (in order to avoid testing in the most acute phase of the illness). The Finnish version of the Hinting Task (Corcoran et al., 1995) was included in the cognitive test battery, containing ten short vignettes involving two people in a conversation. The task is to infer what a person is implying indirectly. In each item, a correct answer gives two points. In case of an incorrect answer, an additional hint is given, after which a correct answer gives one point. Roberts' unpublished additional instruction (2006) was used for scoring. Supplementary Table 2 shows an example of the Hinting Task items.

Other clinical evaluation included the BPRS-E interview (Ventura et al., 1993) and, in the follow-up phases, the SCID-I (First et al., 2002). Level of functioning was assessed with the Global Assessment of Psychosocial Functioning (GAF) (American Psychiatric Association, 2013) on a scale of $0-100$ in every study phase. Retrospective DSM-IV diagnoses were made by a senior psychiatrist (JS) after the 12-month follow-up using all available information including 2- and 12month SCID interviews and medical records. In the analyses, the FEP group was divided into participants with 1) schizophrenia and 2) other psychoses (schizophreniform disorder, 
schizoaffective disorder, bipolar I disorder, major depressive disorder with psychotic features, brief psychotic disorder with psychotic features, and psychotic disorder NOS; Table 1).

\subsection{Statistical analyses}

To take varying Hinting Task item quality and difficulty into account, dichotomized data (scores 01 or 2) were analyzed with Mplus 7.4 (Muthén and Muthén) to obtain a single-dimensional WLSMV factor solution (based on the tetrachoric correlation matrix, as the items were dichotomous). For use as ability scores in further analyses, factor scores were estimated with the maximum a posteriori method (the Mplus default for WLSMV), where the point estimate is the highest point of the distribution on the latent factor. Factor scores were normalized based on the control group distribution.

As recommended by Dunn et al. (2014), we used McDonald's coefficient omega (range 0-1, higher values indicating greater consistency), a measure appropriate for categorical items, to assess the internal consistency (reliability) of the Hinting Task. Omega was estimated with the MBESS R package version 4.4.0 (Kelley, 2007) with bias-corrected and accelerated bootstrap confidence interval (CI) (10 000 bootstrap replications).

A receiver operating curve (ROC) was calculated for the ability of the Hinting Task factor score to distinguish between the control and FEP groups, using pROC R package version 1.10.0 (Robin et al., 2011) with and without the default binormal smoothing, and the associated area under the curve (AUC) was estimated with bootstrap CI (2 000 bootstrap replications).

An inverted square root transformation was used to normalize the scores of Logical Memory delayed recognition. We also transformed Trail Making and Tapping Task performance times to denote speed, by taking the reciprocal, normalizing distributions and making higher values indicate better performance also in these tasks. Other cognitive variables were used as raw scores. For comparing high and low functioning patients, GAF scores were dichotomized into $\geq 50$ points and 
$<50$ points. To summarize cognitive performance, a single exploratory factor model of twenty cognitive test variables was constructed with Mplus using MLR estimation (Supplementary Table 3). Maximum a posteriori factor scores for this $g$ factor were used in regression models as an overall index of general cognitive performance.

All remaining analyses employed IBM SPSS Statistics for Windows, version 24 (IBM Corp., 2016). Groups were compared with the Mann-Whitney $U$-test, due to the skewness of score distributions. Linear regression models were computed with the Hinting Task factor score as the dependent variable, the independent variables in different models being age, gender, $g$ factor, the 2month functioning score, and the dichotomized group variable (control/FEP; control/schizophrenia; control/other psychosis; schizophrenia/other psychosis). Regression effect sizes are expressed as degrees of freedom -adjusted $R^{2}$ values to facilitate comparability between models.

\section{Results}

66 FEP and 62 control participants were included in the study (Table 1). 38 (57.6\%) of the FEP group were inpatients at the time of study enrollment. In the FEP group, 25 (37.9\%) had schizophrenia and the rest were diagnosed with other psychotic disorders. At two months when the cognitive testing was done, $38 \%$ of the FEP participants were in remission (Andreasen et al., 2005) during the past week, and the rest were currently experiencing symptoms at the psychotic level.

\subsection{Overall cognitive performance}

Compared to controls, those with FEP on average performed worse on every cognitive measure, and the difference was also apparent in the $g$ factor (Table 1). 


\subsection{Hinting Task item analysis}

The internal consistency of the Hinting Task calculated from the dichotomized data was modest, with McDonald's categorical omega estimated at 0.57 (95\% CI 0.36, 0.71). In the single dimensional factor solution, items 8 and 9 had the weakest loadings and item 10 the strongest. Items 9 and 10 were the easiest (100\% and 97\%, respectively, of the controls received the full score), and items 1 and 8 were the most difficult (39\% and 47\% correct). Factor scores were moderately skewed (-0.71).

\subsection{Hinting Task group differences}

Figures 1-3 show the scores on the Hinting Task in different groups. Mean (M) raw scores were 16.0 in FEP and 17.4 in controls (Cohen's $d=0.50$ calculated from factor scores; medium effect), with wider variance in the FEP group (Table 1). Due to many near-perfect performances, raw sum scores of the Hinting Task were highly skewed $(-1.7)$.

The smoothed and unsmoothed ROC is presented in Figure 4. The AUC of the unsmoothed curve was $0.63(95 \%$ CI $0.53,0.73)$. The Youden-optimized cut-off yielded a sensitivity of $40 \%$ and a specificity of $85 \%$, approximately corresponding to a sum score of $\geq 15$.

In the FEP group, there were no significant gender differences ( $\mathrm{M}$ for females 16.6, males 15.7), but among the controls, females scored significantly higher on the Hinting Task (M 18.4) than males (M 16.8; Mann-Whitney test $U=233.0, p<0.003)$. Age was not associated with performance on the Hinting Task (Table 2).

\subsection{Regression analyses comparing controls to the whole FEP group}

In linear regression analysis, the group difference between FEP and controls remained significant after controlling for age and gender (Table 3). Adding the group variable to the model improved the explained variance by $6 \%$ (change in adjusted $R^{2}$ ). 
However, when the $g$ factor was controlled for, the group variable did not explain the Hinting Task result. In other words, the difference between FEP and control groups in the ToM task was no longer significant when general cognition was controlled for $\left(R^{2}\right.$ change $\left.-0.4 \%\right)$; adding the $g$ factor weakened the $\beta$ of the group variable by $75 \%$.

Additional exploratory analyses showed that of the individual cognitive tests, the group difference between patients and controls was best explained by WAIS-III Digit Symbol (which explained 54\% of the group difference), WMS-III Logical Memory (delayed recall $43 \%$ and immediate recall 38\%), and WAIS-III Vocabulary (43\%). The percentages reflect the change in Beta coefficient of the group variable when the neurocognitive test is added to the model.

\subsection{Regression analyses comparing controls to the diagnostic groups}

When comparing the Hinting Task performance of the controls to only the patients with schizophrenia (Cohen's $d=1.1$; large effect), adding the $g$ factor into the model improved the explained variance by $8 \%$ (Table 4). The group difference remained significant after controlling for the $g$ factor.

The group difference between controls and those with other psychosis diagnoses was not significant when controlling for age and gender. In this comparison, gender was the best predictor of Hinting Task performance $(\beta=-0.26, p=0.009)$.

\subsection{Diagnostic groups and functioning in the FEP group}

Those with schizophrenia averagely scored lower on the Hinting Task than the other psychosis patients (Cohen's $d=0.56$; medium effect) (Table 1). In a regression model, those with schizophrenia performed worse than the other patients also when controlling for age and gender ( $\beta$ $=-0.29, p=0.020, R^{2}$ change $7 \%$ ). When also controlling for general cognitive performance, the significance of diagnosis attenuated (group $\beta=-0.23, p=0.061, R^{2}$ change $4 \%$ ). 
The level of functioning at the 2-month follow-up was associated with the Hinting Task performance in the FEP group (Table 2). High-functioning patients (GAF score $\geq 50, n=20$ ) performed better on the Hinting Task than those with low functioning $(\mathrm{n}=43)$ (Mann-Whitney $U=$ $666.5, p<0.001)$. The patients with high functioning (M 17.8) did not differ from controls (M 17.4) on the Hinting Task (Mann-Whitney $U=743.0, p=0.183$ ). In regression models, level of functioning as measured by GAF was associated with Hinting Task score $(\beta=0.43, p=0.001)$ when age and gender were controlled for, and this association remained significant after controlling for general cognition (functioning $\beta=0.32, p=0.022$ ).

\section{Discussion}

\subsection{Performance on the Hinting Task}

Young adults with first psychiatric treatment contact for psychosis were tested two months after onset of treatment using the Finnish version of the Hinting Task. The performance in the FEP group was consistent with previous findings (Bertrand et a1., 2007; Corcoran et al., 1995; Ludwig et al., 2017; Thompson et al., 2012). In line with a recent review reporting consistent deficits in verbal ToM in FEP (Healey et al., 2016), the FEP group performed weaker on the Hinting task compared to controls. The results of the control participants were comparable with previous findings, the mean score of 17.4 being at the same level as the previously reported mean scores of $16.8-18.1$ (Bertrand et al., 2007; Ludwig et al., 2017; Pinkham et al., 2016; Thompson et al., 2012). The performance of the controls varied less (standard deviation (SD) 1.6) than that of patients, replicating the findings of the above-mentioned population samples (SD 1.2-1.6). Female controls performed better on the Hinting Task than males, but there was no statistically significant gender difference among patients, which may be due to lack of statistical power in this sample size.

Individuals with FEP were not a homogeneous group in terms of ToM abilities as measured by the Hinting Task. When gender and age were controlled for, Hinting Task performance did not 
significantly differ between controls and those with other psychotic disorders than schizophrenia. In contrast, patients with schizophrenia scored 2 points lower than patients with other psychotic illnesses (M 14.8 versus 16.8) on the Hinting Task. In a previous study, patients with stable schizophrenia or schizoaffective disorder scored even lower, on average 13.6 (Pinkham et al., 2016). Another study using the Finnish version obtained a mean of 14.5 in a sample with mostly schizophrenia patients (Voutilainen et al., 2016), which is very close to our results.

\subsection{Hinting Task performance in relation to level of functioning}

In line with previous studies reporting strong associations between level of functioning and ToM abilities in psychosis (Fett et al., 2011), we found that the best functioning patients performed better than the other patients on the Hinting Task. Their performance in inferring intentions from indirect hints was in fact similar to controls. The fact that their good level of functioning was also showing on the Hinting Task suggests its ecological validity. This result also highlights that, when investigating ToM in psychosis, varying levels of functioning should be considered in participant selection and interpretation of results.

Although ToM is related to overall level of functioning and even though level of functioning often varies by disease phase, meta-analytical evidence shows poorer performance in ToM even in remitted patients (Bora et al.,2009). According to follow-up studies, deficits in ToM appear to be quite stable in the different phases of the disease (Healey et al., 2016). Future studies could further investigate the possibly mediating role of (especially negative) symptoms.

Preyious studies have reported that deficits in social cognition may have a stronger association with social functioning than other cognitive deficiencies or symptomatology (Fett et al., 2011; Ludwig et al., 2017; Pijnenborg et al., 2009), and they might even mediate the association between cognition and functional outcome (Schmidt et al., 2011). Of the various measures assessing social cognition, the Hinting Task significantly relates to social competence and functional outcome 
(Pinkham et al., 2016), although it has been noted that a task requiring third-person judgements might not sufficiently reflect actual everyday interaction situations (Gallagher and Varga, 2015; Revsbech et al., 2016).

\subsection{Hinting Task and general cognitive performance}

It has been suggested that as intact social cognition is rare in the presence of poor neurocognition, general neurocognitive functions may be necessary but not sufficient for social cognitive performance (Fanning et al., 2012). In case of general cognitive decline, deficits in monitoring one's own thought processes and also simulating the mental states of others are therefore also possible.

In the current study, as expected, those with FEP performed, on average, weaker in every cognitive measure compared to controls. The general cognitive performance correlated with Hinting Task performance and largely explained the FEP versus control group difference. As a result, controlling for the general cognitive performance, the difference between FEP and control groups in mentalizing - as assessed with the Hinting Task - was no longer significant. ToM deficits therefore overlapped with general cognitive deficits, telling us that processing social information on the Hinting Task relied on general neurocognitive processes, rather than being independently related to the psychotic disorder.

Of the individual cognitive tests, the Digit Symbol task explained the most of the difference between FEP and controls. As the Digit Symbol task assesses processing speed and executive functioning, it is sensitive to general cognitive impairment. A previous study reported processing speed, as assessed by Digit Symbol task, to be an unique significant contributor to efficient ToM in a FEP sample (Ayesa-Arriola et al., 2016). Other studies have also found the Hinting Task to be associated with executive functioning in FEP (Fernandez-Gonzalo et al., 2014) and in schizophrenia (Greig et al., 2004). However, in these studies, executive functioning was assessed for instance, 
with Trail Making B, a set-switching task that was not among the best predictors of group differences in the current study.

Another central test explaining the variance of Hinting Task performance was Logical Memory. This task measures episodic verbal memory by requiring the participant to hear and recollect stories. It demands rapid processing of information, accurate reconstruction of the events, and verbal production - resembling the Hinting Task. This result suggests that episodic memory is needed to conclude what the other persons mean in their indirect speech. The association between Hinting Task and episodic memory has been reported also previously among individuals with schizophrenia (Greig et al., 2004).

That non-social cognitive decline explained so much of the difference in verbal ToM between the FEP and control groups is not surprising, given the previous findings in FEP (FernandezGonzalo et al., 2014) and schizophrenia samples (Greig et al., 2004). However, when comparing controls to a subgroup of patients with a schizophrenia diagnosis, general cognitive performance accounted for less variance in the Hinting Task; the relationship between schizophrenia diagnosis and Hinting Task remained significant at $p<0.05$ after controlling for general cognitive performance. In other words, in the schizophrenia group, the deficits on the Hinting Task were greater than expected in relation to the general cognitive level. This result is congruent with metaanalytical findings where impairments in ToM remained after controlling for overall intellectual functioning among people with schizophrenia (Sprong et al., 2007).

In the current study, the FEP subgroups with schizophrenia and other psychosis did not differ in neurocognitive functioning, but there was a difference in Hinting Task performance. Possible reasons for the discrepancies between diagnostic subgroups in the current study include differences in symptoms and level of functioning. Compared to the other patients, patients with schizophrenia had lower functioning and more negative symptoms, both of which are associated with ToM. In a meta-analysis with schizophrenia patients, general intellectual deficits contributed to ToM 
impairment only in a stable phase of the disease (outpatients and inpatients just before discharge), leading to a conclusion that in a more acute phase, the relationship between symptoms and ToM may mask the effect of general cognition (Bora 2009). Hence one can speculate if in the current study, more severe symptoms of the patients with schizophrenia masked the association between ToM and neurocognition.

Within the FEP group, the Hinting Task differences between the two diagnostic groups were explained by general cognition. Using the whole FEP group, the level of functioning remained a significant predictor of the Hinting Task performance when general cognitive performance was controlled for. Similarly, in a study conducted among individuals with schizophrenia or schizoaffective disorder, the Hinting Task showed a strong relation to functional outcomes while controlling for other neurocognitive variables (Pinkham et al., 2016).

\subsection{Strengths and weaknesses}

The FEP group in this study consisted of various diagnostic groups, allowing a comparison between schizophrenia and other psychotic disorders. The benefit of studying early psychosis as opposed to more chronic patients is that long-term illness and treatment factors do not affect the social cognition in FEP. The majority of the FEP group was currently having symptoms at the psychotic level. Information on the duration of untreated psychosis was not systematically available for the FEP participants. A matched control group was included in the study.

Most of the individuals with FEP had antipsychotic medication, however, medication is unlikely to explain the deficits in social cognition, as previous studies have reported these deficits also in drug-naïve patients (Behere et al., 2009), in patients with ultra-high risk of psychosis, and in unaffected relatives (Bora and Pantelis, 2013; Van Donkersgoed et al., 2015). A meta-analysis showed that antipsychotic dose was not associated with ToM in schizophrenia (Bora et al., 2009). 
Finally, according to a review, antipsychotic use does not seem to have very promising effects on improving social cognition in schizophrenia (Kucharska-Pietura and Mortimer, 2013).

The internal consistency of the Hinting Task was modest. Instead of the sum score, a factor score of the Hinting Task was therefore used, taking into account the varying difficulty level and relevance of the 10 items. ToM was assessed using the Hinting Task, and our findings are limited to this "mental state reasoning" part of ToM, while other ToM tasks may be associated with symptomatology and cognition in other ways (Fernandez-Gonzalo et al., 2014). A weakness of the Hinting Task is the ceiling effect (Davidson et al., 2017): many participants get a perfect score. To standardize the test and make it more challenging, Roberts' (2006) additional scoring manual was used in the current study. Fairly similar additional criteria, defined by Pinkham et al. (2016), have been used in a previous FEP study (Ludwig et al., 2017).

A previous meta-analysis by Sprong et al. (2007) investigated IQ as possible moderator affecting ToM performance, however, few of the included studies had used the Hinting Task. While some previous studies have examined the overlap between social cognition and neurocognition with factor modelling (Sergi et al., 2007) or correlations (Greig et al., 2004), in the current study, the portion of variance explained by neurocognition was calculated with regression models, and individual tests were also examined. Compared to many previous studies, a relatively broad neuropsychological assessment was administered in our study, allowing for controlling the role of general cognition in verbal ToM. The $g$ factor was most strongly loaded with verbal memory tasks, Trail Making, and Digit Symbol.

Functioning was measured with GAF, a subjective estimation of the level of functioning, which combines functioning and psychiatric symptom severity. Linking the indication of functional deterioration to the severity of psychopathological symptoms, pure functioning without symptoms cannot be separated on this scale. Therefore, low GAF scores may account for deficits in social functioning or severity of symptoms. 


\subsection{Conclusions}

Our study supports previous findings (Bora and Pantelis, 2013) showing deficits in mental state reasoning already in early psychosis. They largely overlap with deficits in general cognitive processes. However, the current study found that in a subgroup of the FEP patients with schizophrenia, specific impairments are present on the Hinting Task, independent from general cognitive deficits. It should also be noted that ToM performance was heterogeneous in the whole FEP group, with a large portion of the FEP group having no deficits in ToM, as measured with the Hinting Task.

Social functioning is strongly associated with social cognition ability, and the efficacy of social cognitive training to ToM measures was recently studied in a review by Kurtz et al. (2016), the effect-sizes being moderate to large. Increasing social skills may enhance social support perceived by the patient, which in turn may prevent relapses and improve well-being in individuals with early psychosis. As cognitive deficits are a potential intervention target in FEP, it is worth pondering whether enhancing ToM should be a specific objective. A previous meta-analysis in nonaffective psychoses (Fett et al.,2011) concluded that social cognition may be an even more important treatment target than neurocognition. Based on our results, this may be true for schizophrenia, but not necessarily for other psychoses, as ToM was better accounted for by general cognition than by psychosis, when the FEP group was investigated as a whole. However, in schizophrenia assessing social cognition already in early stages should be an important part of cognitive assessment in clinical work. 


\section{Acknowledgements}

Helsinki Psychosis Study is a collaboration between the National Institute for Health and Welfare, University Hospital District of Helsinki and Uusimaa, and Aalto University. The authors wish to thank Marjut Grainger, Laura Hietapakka, Sanna Järvinen, Tuula Mononen, Teemu Mäntylä, Eva Rikandi, and all the participants in the study.

\section{Funding}

This study was funded by the European Union's Seventh Framework Programme for project METSY - Neuroimaging platform for characterization of metabolic co-morbidities in psychotic disorders (\# 602478 to JS), the Academy of Finland (\#278171 to JS and \#310295 to MT-H), the Yrjö Jahnsson Foundation (\#6781 to ML), State funding for university-level health research (Hospital District of Helsinki and Uusimaa \#TYH2013332, \#TYH2014228, \#TYH2017128), the Päivikki and Sakari Sohlberg Foundation (ML), the Sigrid Juselius Foundation (JS), and the Finnish Cultural Foundation (JS). The funding organizations played no further part in study design, in the collection, analysis and interpretation of data, or in writing of the paper.

\section{Conflict of Interest}

ML has received financial compensation for an interview from Lundbeck. TM has got rewards for lectures from Lundbeck and Eli Lilly, and TK from Lundbeck and Janssen-Cilag. All other authors declare that they have no conflicts of interest.

\section{Authors' contributions}

JS and TK are principal investigators in the Helsinki Psychosis Study. ML, IH, ST, and MJ designed the analysis plan. ST and ML contributed to designing the cognitive testing. ML and MT$\mathrm{H}$ participated in collecting the data, and JS was responsible for DSM-IV diagnoses. ML, ST, and 
UP undertook the statistical analyses. ML wrote the first draft of the manuscript. All authors contributed to and have approved the final manuscript.

\section{Figure legends}

Figure 1. Population pyramid of Hinting Task raw scores in the FEP and control groups.

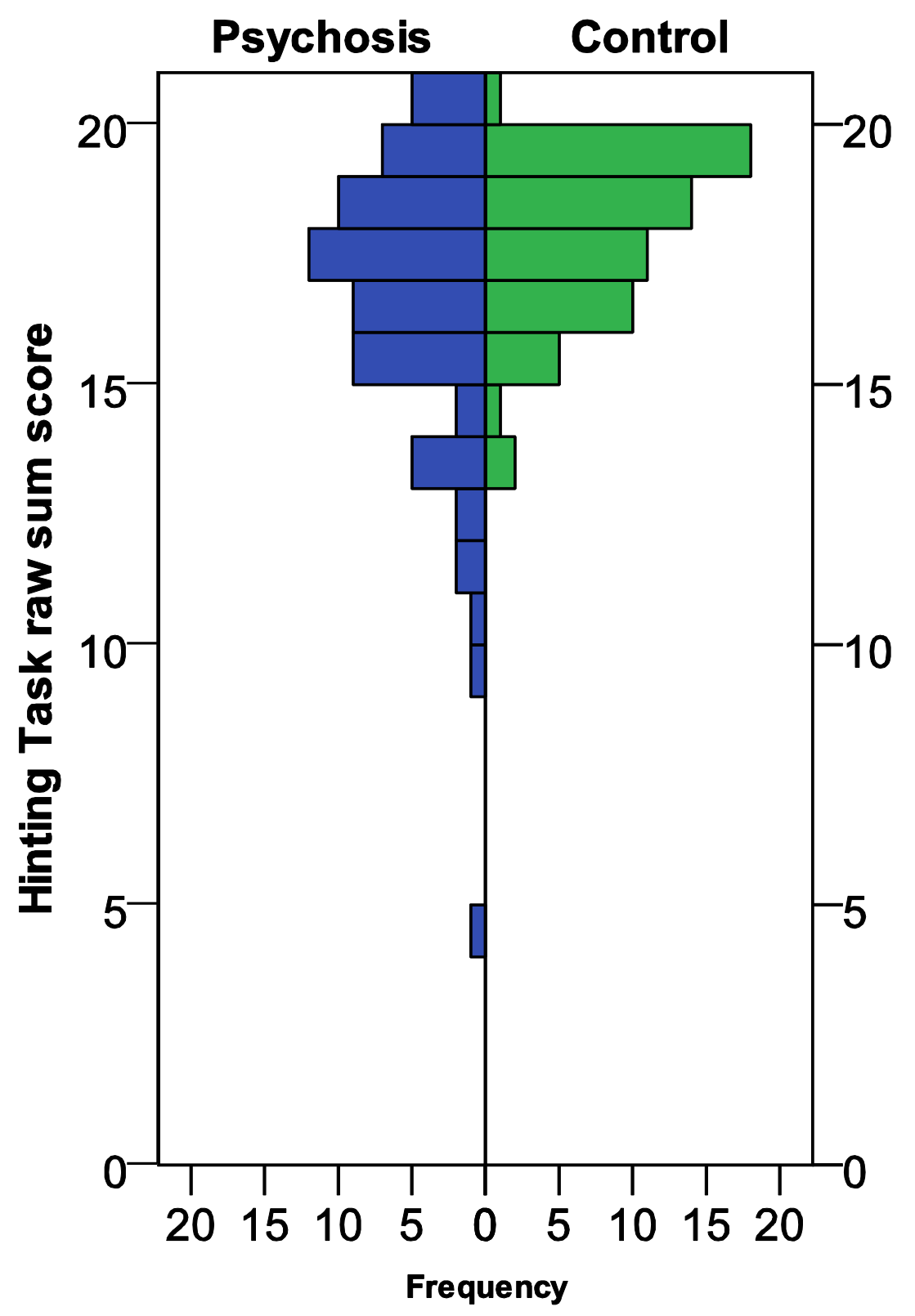

Figure 2. Population pyramid of Hinting Task raw scores in the diagnostic groups. 


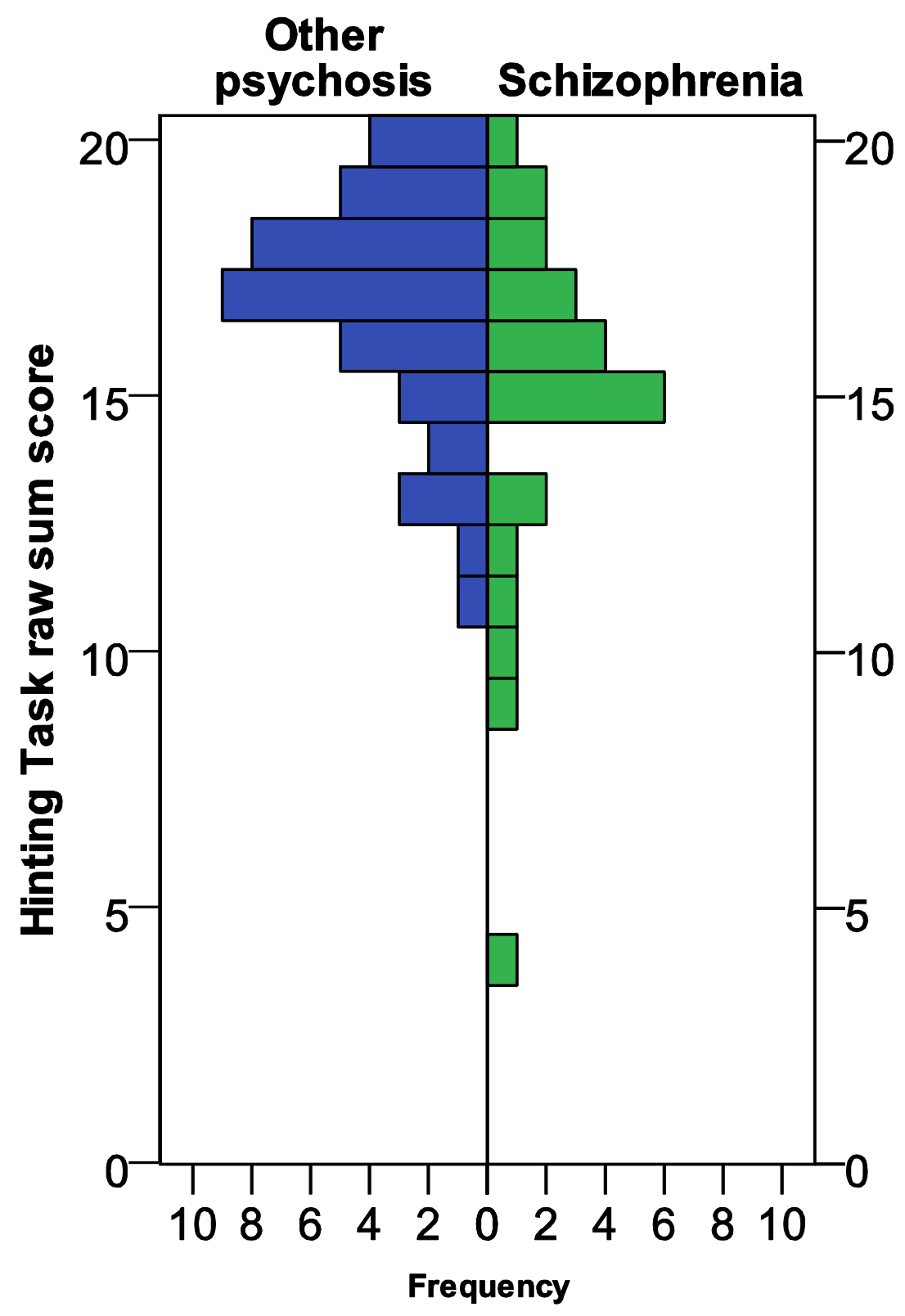

Figure 3. Population pyramid of Hinting Task raw scores in the FEP groups with low and high functioning. 


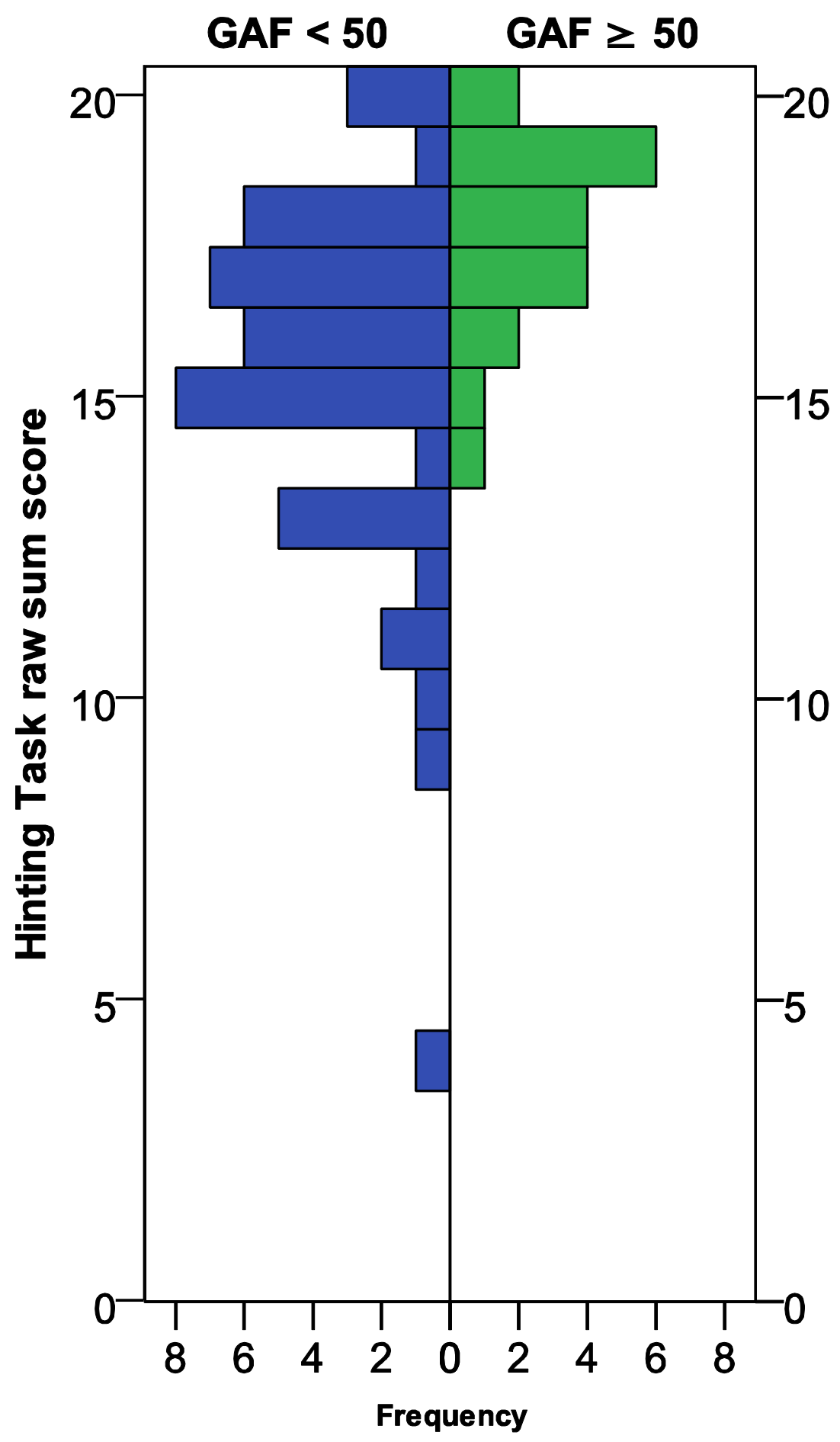

Figure 4. Smoothed (dotted line) and unsmoothed receiver operating curves for distinguishing between FEP and control groups with the Hinting Task factor score. 


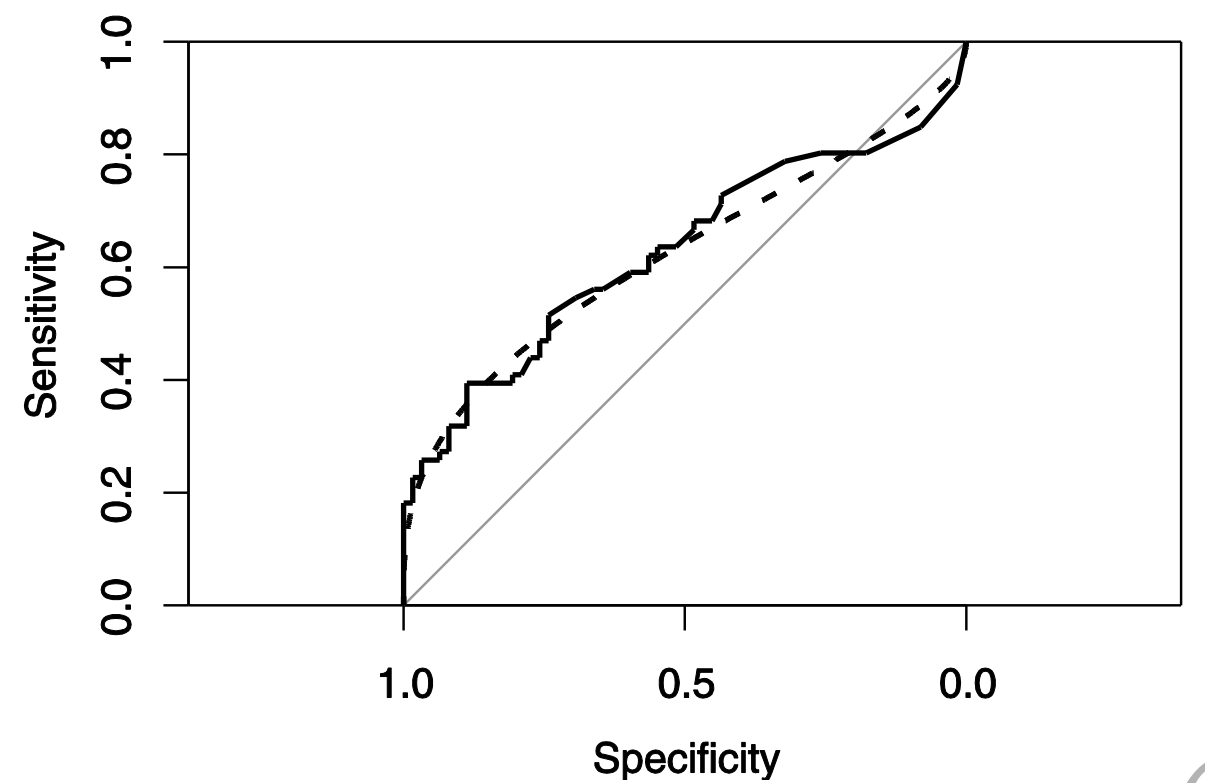




\section{Tables}

Table 1. The demographic information and raw scores of cognitive tests in the participant groups. Frequency (\%) or M (SD), range

\begin{tabular}{|c|c|c|c|c|c|c|}
\hline & FEP, $n=66$ & Controls, $n=62$ & $\begin{array}{l}\text { Group } \\
\text { difference }^{\text {a }}\end{array}$ & $\begin{array}{l}\text { FEP schizophrenia, } \\
n=25\end{array}$ & $\begin{array}{l}\text { FEP other } \\
\text { psychosis }^{\text {b }}, n=41\end{array}$ & $\begin{array}{l}\text { Group } \\
\text { difference }\end{array}$ \\
\hline Male & $41(62.1 \%)$ & $41(66.1 \%)$ & $p=0.637$ & $15(60.0 \%)$ & $26(63.4 \%)$ & $p=0.781$ \\
\hline Age & $\begin{array}{l}26.6(5.6), 18.4- \\
41.3\end{array}$ & $\begin{array}{l}26.3(5.9), 19.1- \\
43.8\end{array}$ & $p=0.564$ & $\begin{array}{l}26.2(5.7), 18.4- \\
41.3\end{array}$ & $\begin{array}{l}26.8(5.6), 18.4- \\
39.3\end{array}$ & $p=0.547$ \\
\hline Years of education $^{c}$ & $\begin{array}{l}14.1(3.3), 9.5- \\
23.5\end{array}$ & $\begin{array}{l}14.8(2.4), 11.0- \\
22.0\end{array}$ & $p=0.032$ & $13.2(3.1), 9.5-22.0$ & $\begin{array}{l}14.7(3.3), 10.0- \\
23.5\end{array}$ & $p=0.055$ \\
\hline GAF baseline & $37.5(7.3), 30-65$ & $83.2(9.0), 55-95$ & $p<0.001$ & $34.2(3.7), 30-40$ & $39.4(8.3), 30-65$ & $p=0.006$ \\
\hline GAF 2 months & 44.2 (10.9), $25-75$ & - & - & $38.0(9.8), 25-75$ & $48.2(9.7), 32-70$ & $p<0.001$ \\
\hline $\begin{array}{l}\text { High functioning ( } 2 \\
\text { month GAF } \geq 50 \text { ) }\end{array}$ & $20(30.3 \%)$ & - & & $2(8.0 \%)$ & $18(43.9 \%)$ & $p=0.001$ \\
\hline Hinting Task score & $16.0(3.0), 4-20$ & $17.4(1.6), 13-20$ & & $14.8(3.6), 4-20$ & $16.8(2.3), 11-20$ & $p=0.016$ \\
\hline below 13 & $7(10.6 \%)$ & 0 & & $5(20.0 \%)$ & $2(4.9 \%)$ & \\
\hline $13-15$ & $16(24.2 \%)$ & $8(12.9 \%)$ & & $8(32.0 \%)$ & $8(19.5 \%)$ & \\
\hline $16-18$ & $31(47.0 \%)$ & $35(56.5 \%)$ & & $9(36.0 \%)$ & $22(53.4 \%)$ & \\
\hline $19-20$ & $12(18.2 \%)$ & $19(30.6 \%)$ & & $3(12.0 \%)$ & $9(22.0 \%)$ & \\
\hline Hinting Task factor score & $-0.7(1.7),-4.9-1.9$ & $0.0(1.0),-2.6-1.9$ & $p=0.012$ & $-1.3(1.7),-4.9-1.9$ & $-0.4(1.6),-3.9-1.9$ & $p=0.028$ \\
\hline Block Design & 44.8 (13.2), 12-65 & $50.9(12.3), 11-$ & $p=0.007$ & 44.3 (13.7), 12-64 & $45.1(13.0), 12-65$ & $p=0.884$ \\
\hline Vocabulary & 43.9 (10.6), 22-64 & $48.7(9.6), 22-64$ & $p=0.011$ & $41.6(11.8), 22-62$ & $45.3(9.6), 26-64$ & $p=0.303$ \\
\hline Digit Symbol & 64.8 (14.6), 32-105 & $79.9(12.7), 54-$ & $p<0.001$ & 61.3 (18.7), 32-105 & $66.9(11.2), 43-89$ & $p=0.084$ \\
\hline $\begin{array}{l}\text { Logical Memory, } \\
\text { immediate }\end{array}$ & & $45.1(9.7), 17-63$ & $p<0.001$ & $35.2(12.1), 17-62$ & 38.3 (10.6), 3-60 & $p=0.159$ \\
\hline Logical Memory, delayed & $22.5(8.8), 4-44$ & $28.9(7.0), 12-44$ & $p<0.001$ & $21.4(9.8), 7-42$ & $23.2(8.2), 4-44$ & $p=0.285$ \\
\hline $\begin{array}{l}\text { Logical Memory, delayed } \\
\text { recognition }\end{array}$ & $25.4(3.1), 17-30$ & $26.6(2.3), 17-30$ & $p=0.028$ & $25.1(3.1), 17-30$ & $25.6(3.1), 17-30$ & $p=0.368$ \\
\hline Letter-number Sequencing & $9.7(2.5), 5-17$ & $10.8(2.3), 6-18$ & $p=0.004$ & $9.0(2.0), 5-13$ & $10.1(2.6), 5-17$ & $p=0.072$ \\
\hline Spatial Span & $17.9(3.5), 8-26$ & $19.3(2.6), 13-26$ & $p=0.007$ & $17.4(3.3), 10-23$ & $18.2(3.6), 8-26$ & $p=0.577$ \\
\hline Word List, immediate & $31.5(6.1), 17-44$ & - & - & $29.9(6.4), 17-43$ & $32.4(5.9), 20-44$ & $p=0.137$ \\
\hline Word List, long delay & $6.9(3.2), 0-12$ & - & - & $6.3(3.5), 0-12$ & $7.3(3.0), 1-12$ & $p=0.306$ \\
\hline
\end{tabular}


Word List, delayed

recognition

Visual Reproduction, immediate

Visual Reproduction, delayed

Trail Making A (s)

Trail Making B (s)

Trail Making C

(alphabets) (s)

Verbal Fluency, animals

Verbal Fluency, letter s

Verbal Fluency, letter $\mathrm{k}$

CPT-IP d-prime

Tapping dominant hand $\mathrm{M}$

(ms)

Tapping nondominant

hand $\mathrm{M}(\mathrm{ms})$

$g$ factor

$\mathrm{M}$, mean

$\mathrm{SD}$, standard deviation

FEP, First-episode psychosis

GAF, Global Assessment of Functioning

CPT-IP, Continuous Performance Test - Identical Pairs

${ }^{\text {a }}$ Mann-Whitney $U$-test or Pearson Chi-Square test. Statistically significant results shown in bold.

${ }^{\mathrm{b}}$ Other psychotic disorders include Schizophreniform disorder ( $\left.\mathrm{n}=14\right)$, Psychotic disorder NOS (n=12), Bipolar I disorder ( $\mathrm{n}=6$ ), Schizoaffective disorder ( $n=3)$, Major depressive disorder with psychotic features $(n=3)$, and Brief psychotic disorder with psychotic features $(n=3)$.

${ }^{\mathrm{c}}$ Self-reported full-time education

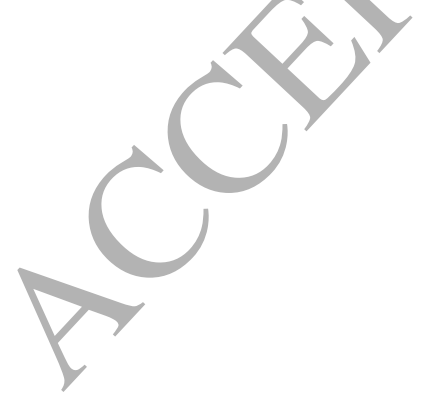


Table 2. Nonparametric Spearman correlations between the Hinting Task raw scores and study covariates in first-episode psychosis group and controls

\begin{tabular}{lccccc}
\hline Group & Age & $\begin{array}{c}\text { Years of } \\
\text { education }\end{array}$ & $g$ factor & GAF baseline & GAF 2 months \\
\hline FEP, $n=66$ & 0.00 & $0.26^{*}$ & $0.34^{* *}$ & 0.16 & $0.46^{* *}$ \\
Controls, $n=62$ & 0.10 & 0.24 & $0.34^{* *}$ & 0.03 & - \\
\hline
\end{tabular}

${ }^{\mathrm{a}}$ Self-reported full-time education

GAF, Global Assessment of Functioning

FEP, First-episode psychosis

$* p<.05, * * p<.01$

Table 3. Performance on the Hinting Task in FEP and control groups. Regression coefficients $(\beta)$ and the significance of the group variable $(p)$, and adjusted $R^{2}$ of the models. Reference categories in parentheses.

\begin{tabular}{llll}
\hline Predictors in the model & $\beta$ (group) & $p$ & $R^{2}$ \\
\hline Gender (male) + age & & & 0.02 \\
Gender (male) + age + group (FEP) & 0.259 & $\mathbf{0 . 0 0 3}$ & 0.08 \\
& & & \\
Gender (male) + age + $g$ factor & & & 0.16 \\
Gender (male) + age + $g$ factor + group (FEP) & 0.064 & 0.518 & 0.16 \\
\hline
\end{tabular}

FEP, First-episode psychosis

Table 4. Performance on the Hinting Task in two diagnostic groups and controls. Regression coefficients $(\beta)$ and the significance of the group variable $(p)$, and adjusted $R^{2}$ of the models. Reference categories in parentheses.

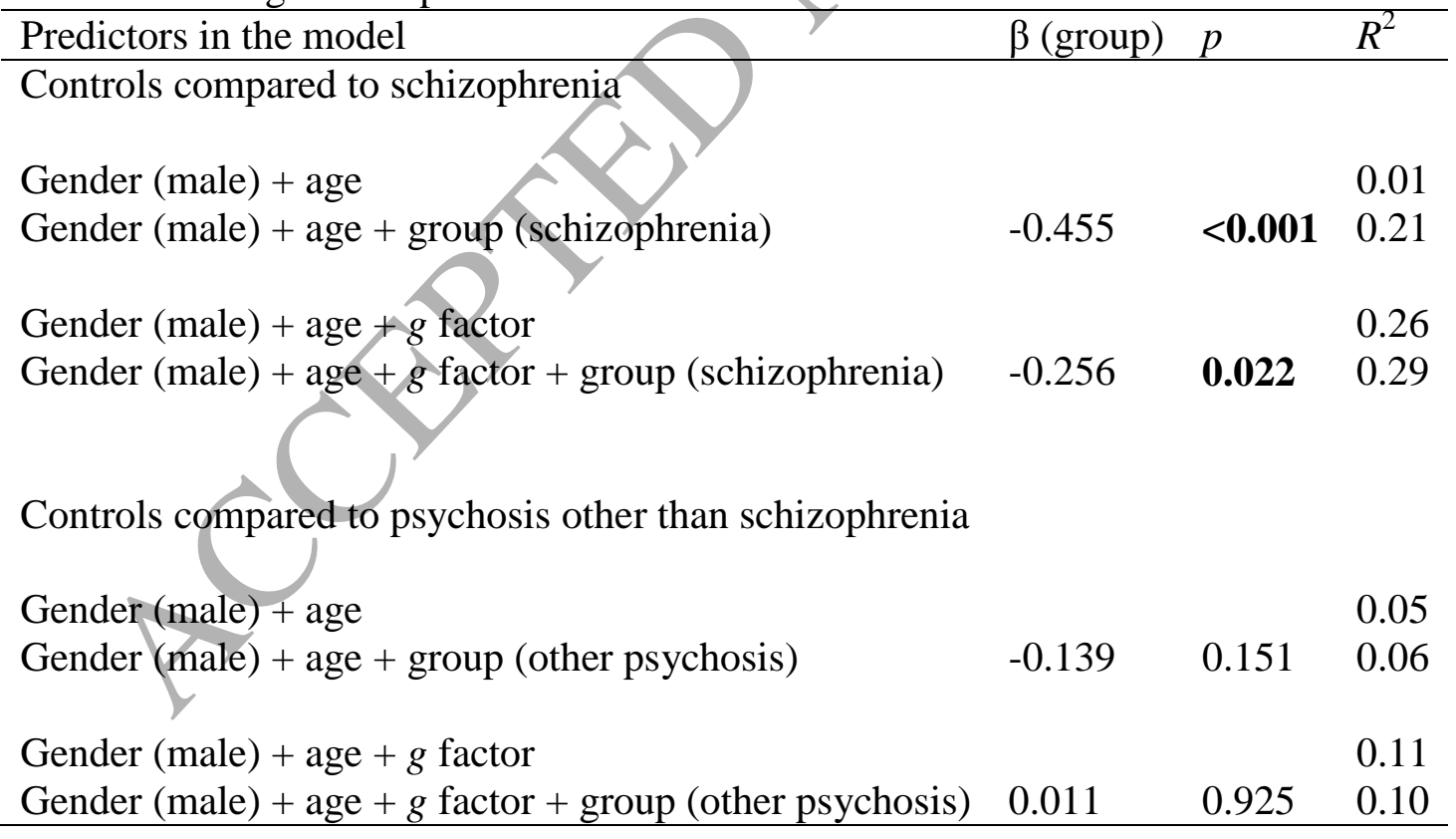




\section{References}

Addington, J., Girard, T.A., Christensen, B.K., Addington, D., 2010. Social cognition mediates illness-related and cognitive influences on social function in patients with schizophreniaspectrum disorders. J. Psychiatry Neurosci. 35, 49-54.

American Psychiatric Association, 2013. Diagnostic and Statistical Manual of Mental Disorders. American Psychiatric Publishing, Arlington, VA.

Andreasen, N.C., Carpenter Jr, W.T., Kane, J.M., Lasser, R.A., Marder, S.R., Weinberger, D.R., 2005. Remission in schizophrenia: proposed criteria and rationale for consensus. Am. J. Psychiatry 162, 441-449.

Ayesa-Arriola, R., Setien-Suero, E., Neergaard, K.D., Ferro, A., Fatjo-Vilas, M., Rios-Lago, M., et al., 2016. Evidence for trait related theory of mind impairment in first episode psychosis patients and its relationship with processing speed: a 3 year follow-up study. Front. Psychol. 7, 592.

Behere, R. V, Venkatasubramanian, G., Arasappa, R., Reddy, N., Gangadhar, B.N., 2009. Effect of risperidone on emotion recognition deficits in antipsychotic-naive schizophrenia: a short-term follow-up study. Schizophr. Res. 113, 72-76.

Bentall, R.P., de Sousa, P., Varese, F., Wickham, S., Sitko, K., Haarmans, M., et al., 2014. From adversity to psychosis: pathways and mechanisms from specific adversities to specific symptoms. Soc. Psychiatry Psychiatr. Epidemiol. 49, 1011-1022.

Bertrand, M.C., Sutton, H., Achim, A.M., Malla, A.K., Lepage, M., 2007. Social cognitive impairments in first episode psychosis. Schizophr. Res. 95, 124-133.

Bora, E., Eryavuz, A., Kayahan, B., Sungu, G., Veznedaroglu, B., 2006. Social functioning, theory of mind and neurocognition in outpatients with schizophrenia; mental state decoding may be a better predictor of social functioning than mental state reasoning. Psychiatry Res. 145, 95-103.

Bora, E., Pantelis, C., 2013. Theory of mind impairments in first-episode psychosis, individuals at ultra-high risk for psychosis and in first-degree relatives of schizophrenia: systematic review and meta-analysis. Schizophr. Res. 144, 31-36.

Bora, E., Yucel, M., Pantelis, C., 2009. Theory of mind impairment in schizophrenia: meta-analysis. Schizophr. Res. 109, 1-9.

Brüne, M., 2005. "Theory of mind" in schizophrenia: a review of the literature. Schizophr. Bull. 31, $21-42$.

Corcoran, R., Mercer, G., Frith, C.D., 1995. Schizophrenia, symptomatology and social inference: investigating "theory of mind" in people with schizophrenia. Schizophr. Res. 17, 5-13.

Davidson, C.A., Lesser, R., Parente, L.T., Fiszdon, J.M., 2017. Psychometrics of social cognitive measures for psychosis treatment research. Schizophr. Res. Jun 22. pii: S0920-9964(17)303572. doi: 10.1016/j.schres.2017.06.018. [Epub ahead of print]

Dunn, T.J., Baguley, T., Brunsden, V., 2014. From alpha to omega: A practical solution to the pervasive problem of internal consistency estimation. Br. J. Psychol. 105, 399-412.

Fanning, J.R., Bell, M.D., Fiszdon, J.M., 2012. Is it possible to have impaired neurocognition but good social cognition in schizophrenia? Schizophr. Res. 135, 68-71.

Fernandez-Gonzalo, S., Jodar, M., Pousa, E., Turon, M., Garcia, R., Rambla, C.H., et al., 2014. Selective effect of neurocognition on different theory of mind domains in first-episode psychosis. J. Nerv. Ment. Dis. 202, 576-582.

Fett, A.K., Viechtbauer, W., Dominguez, M.D., Penn, D.L., van Os, J., Krabbendam, L., 2011. The relationship between neurocognition and social cognition with functional outcomes in schizophrenia: a meta-analysis. Neurosci. Biobehav. Rev. 35, 573-588.

First, M.B., Spitzer, R.L., Gibbon, M., Williams, J.B.W., 2002. Structured Clinical Interview for DSM-IV-TR Axis I disorders, Research version, Patient edition. (SCID-I/P). Biometrics Research, New York State Psychiatric Institute, New York, NY. (Revision: January 2007). 
Gallagher, S., Varga, S., 2015. Social cognition and psychopathology: a critical overview. World Psychiatry 14, 5-14.

Green, M.F., Horan, W.P., Lee, J., 2015. Social cognition in schizophrenia. Nat. Rev. 16, 620-631.

Green, M.F., Kern, R.S., Braff, D.L., Mintz, J., 2000. Neurocognitive deficits and functional outcome in schizophrenia: are we measuring the "right stuff"? Schizophr. Bull. 26, 119-136.

Green, M.F., Penn, D.L., Bentall, R., Carpenter, W.T., Gaebel, W., Gur, R.C., et al., 2008. Social cognition in schizophrenia: an NIMH workshop on definitions, assessment, and research opportunities. Schizophr. Bull. 34, 1211-1220.

Greig, T.C., Bryson, G.J., Bell, M.D., 2004. Theory of mind performance in schizophrenia: diagnostic, symptom, and neuropsychological correlates. J. Nerv. Ment. Dis. 192, 12-18.

Healey, K.M., Bartholomeusz, C.F., Penn, D.L., 2016. Deficits in social cognition in first episode psychosis: A review of the literature. Clin. Psychol. Rev. Dec;50:108-137.

IBM Corp., 2016. IBM SPSS Statistics for Windows, Version 24.0. Author, Armonk, NY.

Keinänen, J., Mantere, O., Kieseppä, T., Mäntylä, T., Torniainen, M., Lindgren, M., et al., 2015. Early insulin resistance predicts weight gain and waist circumference increase in first-episode psychosis--A one year follow-up study. Schizophr. Res. 169, 458-463.

Kelley, K., 2007. Methods for the behavioral, educational, and social sciences: an R package. Behav. Res. Methods 39, 979-984.

Kucharska-Pietura, K., Mortimer, A., 2013. Can antipsychotics improve social cognition in patients with schizophrenia? CNS Drugs 27, 335-343.

Kurtz, M.M., Gagen, E., Rocha, N.B.F., Machado, S., Penn, D.L., 2016. Comprehensive treatments for social cognitive deficits in schizophrenia: A critical review and effect-size analysis of controlled studies. Clin. Psychol. Rev. 43, 80-89.

Lindgren, M., Mäntylä, T., Rikandi, E., Torniainen-Holm, M., Morales-Muñoz, I., Kieseppä, T., et al., 2017. Childhood adversities and clinical symptomatology in first-episode psychosis. Psychiatry Res. Dec;258:374-381.

Ludwig, K.A., Pinkham, A.E., Harvey, P.D., Kelsven, S., Penn, D.L., 2017. Social cognition psychometric evaluation (SCOPE) in people with early psychosis: A preliminary study. Schizophr. Res. 190, 136-143.

McGlade, N., Behan, C., Hayden, J., O'Donoghue, T., Peel, R., Haq, F., et al., 2008. Mental state decoding $\mathrm{v}$. mental state reasoning as a mediator between cognitive and social function in psychosis. Br. J. Psychiatry 193, 77-78.

Muthén, L.K., Muthén, B.O., 1998-2012. Mplus user's guide. Seventh edition. Los Angeles, CA: Muthén \& Muthén.

Pijnenborg, G.H., Withaar, F.K., Evans, J.J., van den Bosch, R.J., Timmerman, M.E., Brouwer, W.H., 2009. The predictive value of measures of social cognition for community functioning in schizophrenia: implications for neuropsychological assessment. J. Int. Neuropsychol. Soc. $15,239-247$.

Pinkham, A.E., Penn, D.L., Green, M.F., Harvey, P.D., 2016. Social cognition psychometric evaluation: Results of the initial psychometric study. Schizophr. Bull. 42, 494-504.

Revsbech, R., Mortensen, E.L., Nordgaard, J., Jansson, L.B., Saebye, D., Flensborg-Madsen, T., et al., 2016. Exploring social cognition in schizophrenia. Eur. Arch. Psychiatry Clin. Neurosci. $1-9$.

Robin, X., Turck, N., Hainard, A., Tiberti, N., Lisacek, F., Sanchez, J.C., et al., 2011. pROC: an open-source package for $\mathrm{R}$ and $\mathrm{S}+$ to analyze and compare ROC curves. BMC Bioinformatics $12,77$.

Schmidt, S.J., Mueller, D.R., Roder, V., 2011. Social cognition as a mediator variable between neurocognition and functional outcome in schizophrenia: empirical review and new results by structural equation modeling. Schizophr. Bull. 37 Suppl 2, S41-54.

Sergi, M.J., Rassovsky, Y., Widmark, C., Reist, C., Erhart, S., Braff, D.L., et al., 2007. Social 
cognition in schizophrenia: Relationships with neurocognition and negative symptoms. Schizophr. Res. 90, 316-324.

Sprong, M., Schothorst, P., Vos, E., Hox, J., van Engeland, H., 2007. Theory of mind in schizophrenia: meta-analysis. Br. J. Psychiatry 191, 5-13.

Thompson, A., Papas, A., Bartholomeusz, C., Allott, K., Amminger, G.P., Nelson, B., et al., 2012. Social cognition in clinical "at risk" for psychosis and first episode psychosis populations. Schizophr. Res. 141, 204-209.

Van Donkersgoed, R.J.M., Wunderink, L., Nieboer, R., Aleman, A., Pijnenborg, G.H.M., 2015. Social cognition in individuals at ultra-high risk for psychosis: A meta-analysis. PLoS One Oct 28;10(10):e0141075.

Ventura, J., Green, M., Shaner, A., Liberman, R.P., 1993. Training and quality assurance on the BPRS: "the drift busters". Int J Methods Psychiatr Res 3, 221-224.

Voutilainen, G., Kouhia, T., Roberts, D.L., Oksanen, J., 2016. Social Cognition and Interaction Training (SCIT) for adults with psychotic disorders: a feasibility study in Finland. Behav. Cogn. Psychother. 1-6. 